\title{
Improvement in Capacity and Signal Strength using LMS Algorithm
}

\author{
Ruchi Mittal \\ Asstt. Professor \\ LIMAT \\ Faridabad
}

\author{
Kiranpreet Kaur \\ Asstt. Professor \\ BBSBEC \\ Fatehgarhsahib
}

\author{
Magandeep Kaur \\ Lecturer \\ LIMAT \\ Faridabad
}

\begin{abstract}
This paper provides the information regarding the improvement in capacity and signal strength with the implementation of adaptive beamfoming algorithms (LMS and SMI) in adaptive antenna arrays.In this a beamforming algorithm is analyzed for flexible side lobe adjustment. The algorithm places a very low side lobe level over a large width in the beam pattern. The convergence of this algorithm is fast and requires only two to three iterations.
\end{abstract}

\section{Keywords}

Beam forming, covariance matrix, chebychev antenna pattern.

\section{INTRODUCTION}

\section{Adaptive Beam forming using LMS Algorithm}

The LMS algorithm discussed in the previous section is a continuously adaptive algorithm and has a slow convergence when the eigen values of the covariance matrix are widespread. When the transmission is discontinuous, a block adaptive approach would give a better performance than a continuous approach. One such algorithm is the Sample Matrix Inversion (SMI) as shown in fig. (7), which provides good performance in a discontinuous traffic. However, it requires that the number of interferers and their positions remain constant during the duration of the block acquisition.

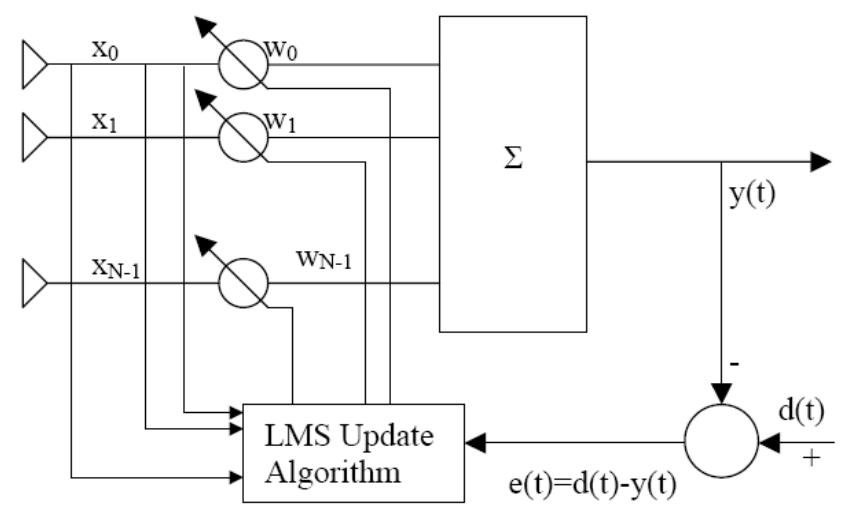

Fig.1. LMS adaptive beam forming network

\section{LMS Formulation:}

The LMS algorithm has a faster convergence rate since it employs direct inversion of the covariance matrix R. Let us recall the equations for the covariance matrix $R$ and the correlation matrix $r$.

$$
\begin{aligned}
R & =E\left[x(t) x^{H}(t)\right] \\
r & =E[d(t) x(t)]
\end{aligned}
$$

If a priori information about the desired and the interfering signals is known, then the optimum weights can be calculated directly by using the Weiner solution,

$$
\mathbf{W}_{\text {opt }}=\mathbf{R}^{-1} \mathbf{r}
$$

However, in practice signals are not known and the signal environment keeps changing. Therefore obtaining the estimates of the covariance matrix $\mathrm{R}$ and the correlation matrix $\mathrm{r}$ can compute optimal weights, by time averaging from the block of input data. The estimates of the matrices over a block size $\mathrm{N}_{2}-\mathrm{N}_{1}$-are given by

$$
\begin{gathered}
\hat{R}=\sum_{i=N_{1}}^{N_{2}} x(i) x^{H}(i) \\
\hat{r}=\sum_{i=N 1}^{N 2} d^{*}(\mathrm{i}) x^{H}(\mathrm{t})
\end{gathered}
$$

Where $\mathrm{i}$ form the lower and the upper limit of the observation interval. The weight vector can now be estimated by the following equation: $\hat{w}=\hat{R}^{-1} \hat{r}$

Based on the above discussion the weights will be updated for each incoming block. There is always a residual error in the SMI algorithm since it is based on estimation. This error is usually greater when compared to the LMS error. The error due to estimates can be computed by the following equation:

$$
\text { 1. } e=\hat{R} w_{o p t}-\hat{r}
$$

The stability of the LMSalgorithm depends on the ability to invert the large covariance matrix. In order to avoid a singularity of the covariance matrix, a zero- mean white Gaussian noise is added to the array response vector. It creates a strong additive component to the diagonal of the matrix. In the absence of noise in the system, a singularity occurs when the number of signals to be resolved is less than the number of 
elements in the array.Since SMI/LMS employs direct matrix inversion the convergence of this algorithm is much faster compared to the LMS algorithm. However, huge matrix inversions lead to computational complexities that cannot be easily overcome.

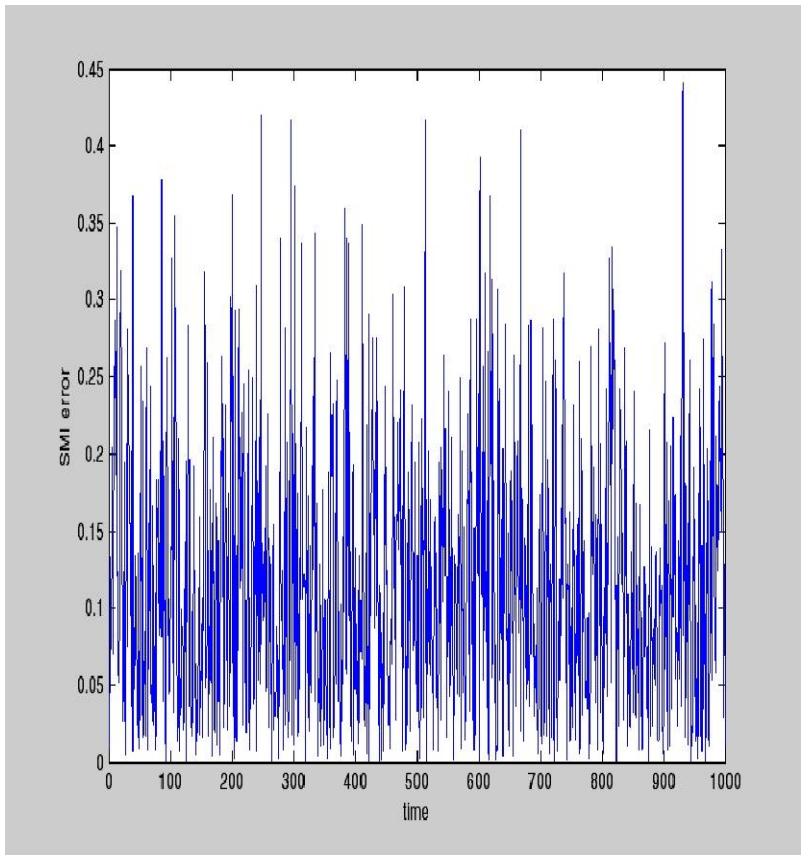

Fig.1: SMI Error Vs Time (blocksize=10,'constant')

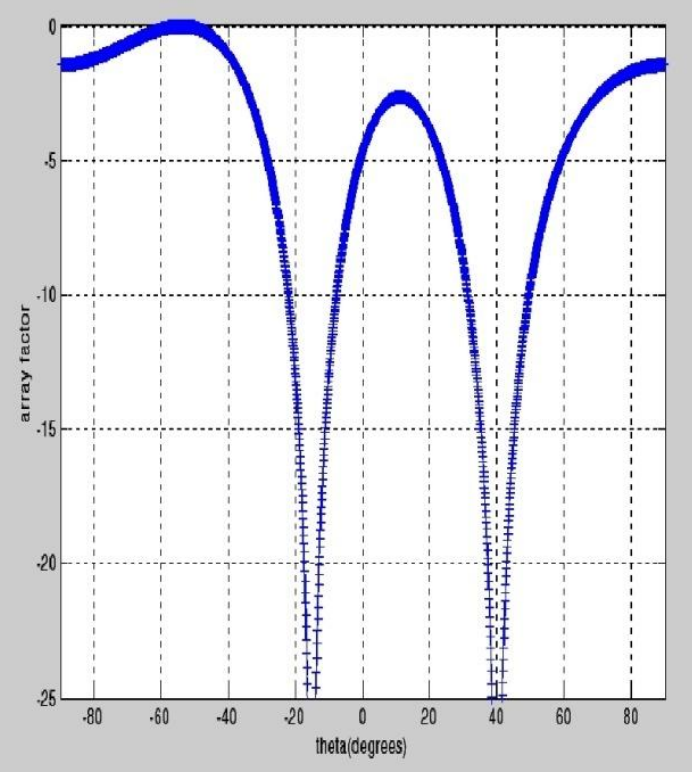

Fig.2: Array Factor vs theta

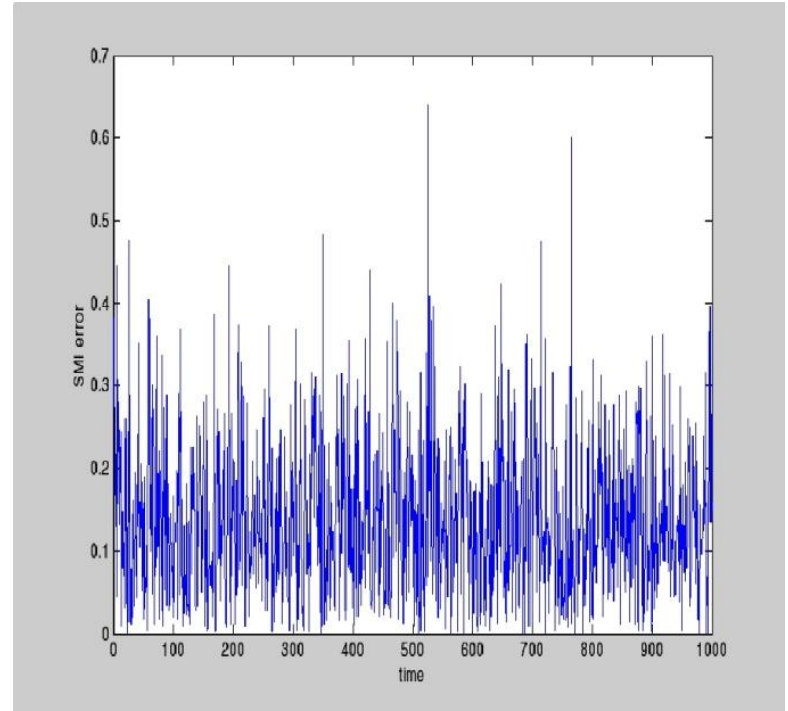

Fig.2: Error Vs. Time (block size=100,'constant')

\section{SIMULATION RESULTS}

The convergence of the proposed algorithm is proved, by synthesizing the Chebyshev antenna pattern, as it is the only and finest example of optimum beam forming with equal side lobe levels for a minimum beam width. This method is applied to uniform linear array for different number of elements and the beam pattern is finally converged to Chebyshev pattern. The uniform linear array pattern is known to have minimum beamwidth (BW), and the finally converged beam also has minimum beamwidth for a given sidelobe level, which is similar to the Chebyshev beam pattern.

1. (a) No of elements in array $=10$, Error $=0.05$,

No of iteration required $=2$,

Total time taken $=20.6$ seconds
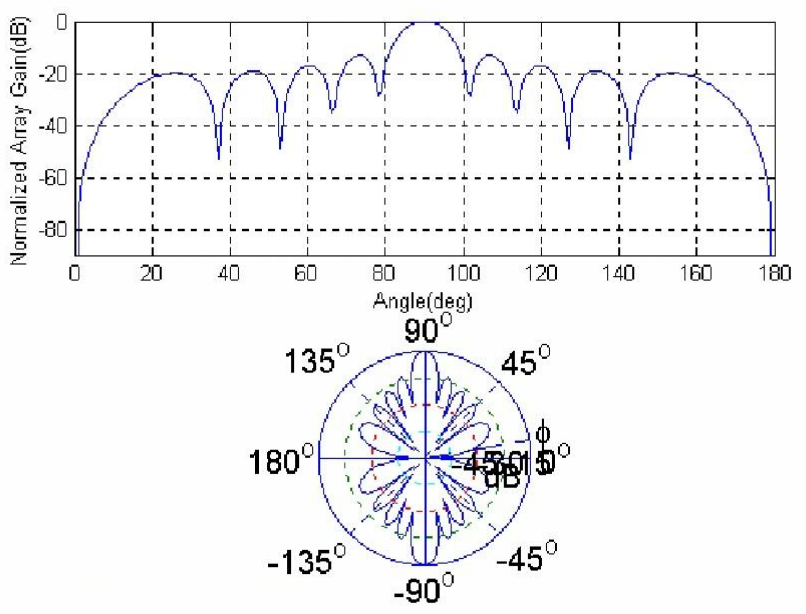

Fig. 1(a) pattern of linear array for $N=10, d=\lambda / 2$ 

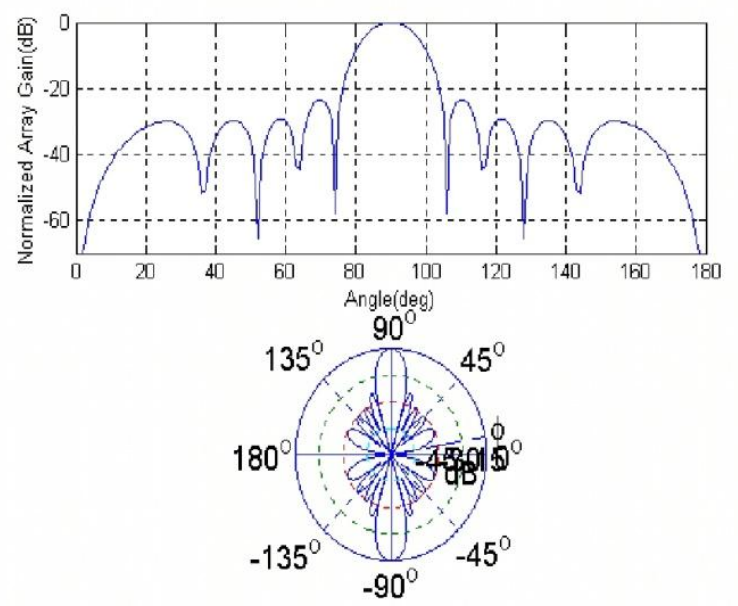

Fig1 (b): Pattern of linear array for $N=10, d=\lambda / 2$ after $1^{\text {st }}$ iteration.
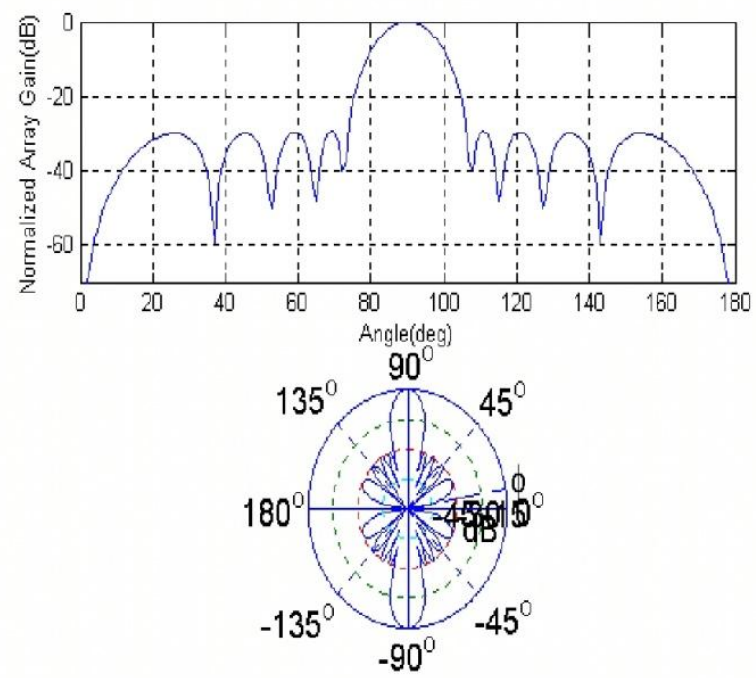

Fig1 (c): Pattern of linear array for $\mathrm{N}=10, \mathrm{~d}=\lambda / 2$ after 2nd iteration.

(b) No of elements in array $=10$, Error $=0.005$,

No of iteration required $=3$

Total time taken $=28.78$ seconds
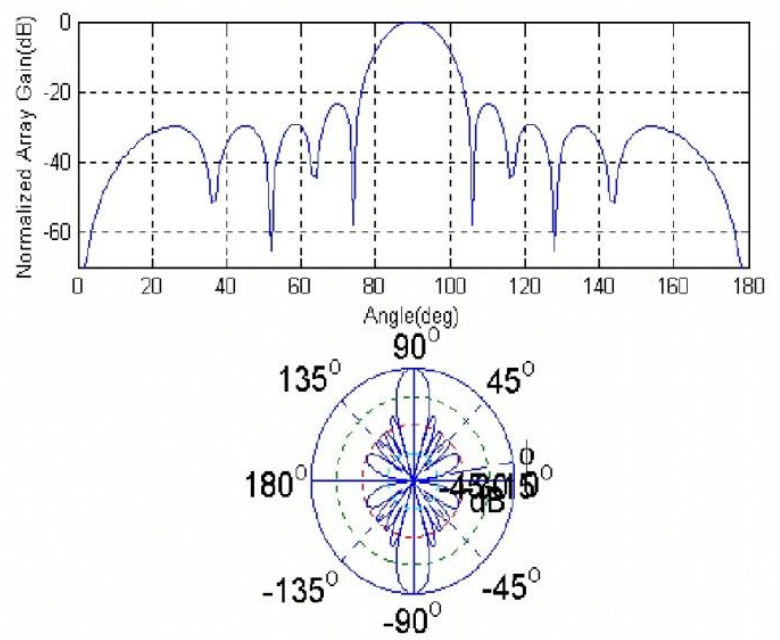

Fig.2 (a): Pattern of linear array for $\mathrm{N}=10, \mathrm{~d}=\lambda / 2$ after 1st iteration.

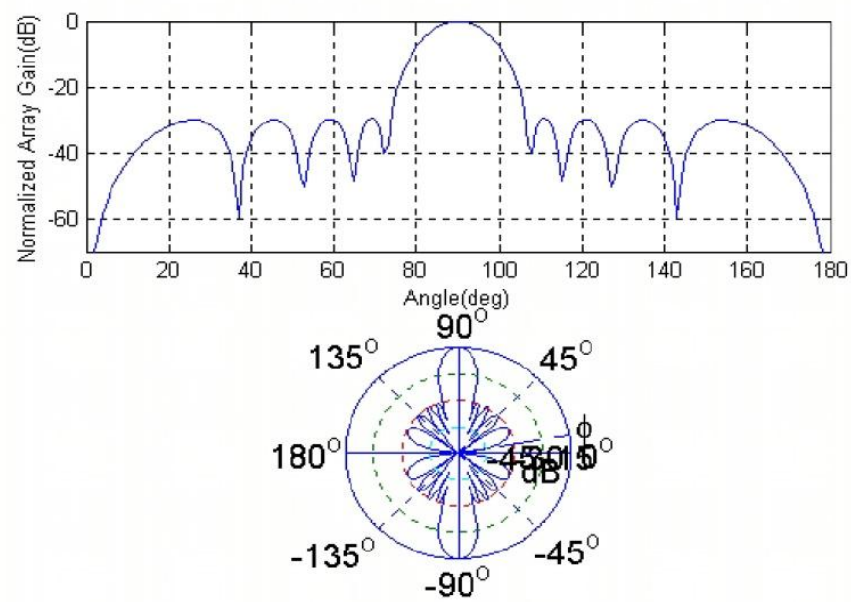

Fig. 2(b): Pattern of linear array for $N=10, d=\lambda / 2$ after 2nd iteration.

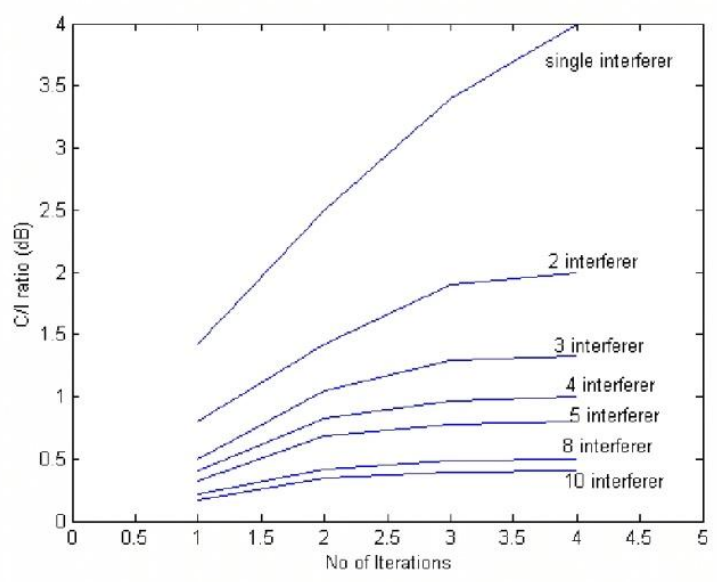

Fig.3: Graph showing increase in CIR ratio for different number of interferers by proposed algorithm 


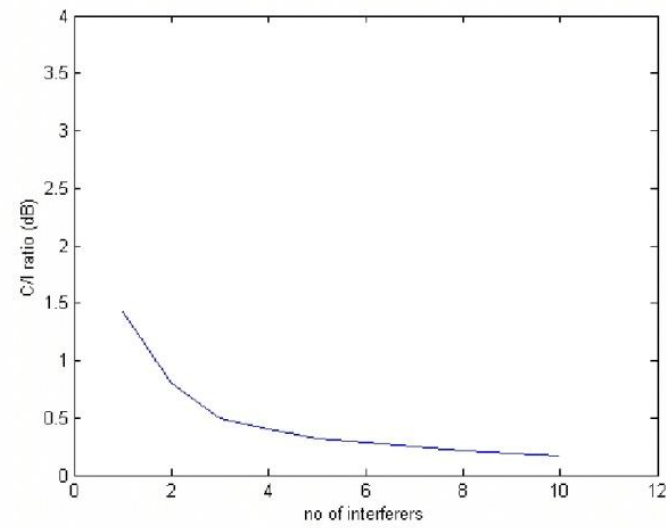

Fig. 4 Graph showing the decrease in CIR as number of interferers increases.

\section{CONCLUSION}

A new array pattern synthesis procedure is analyzed in which minimum mean square error is assigned externally and iterations continue and in each iteration sidelobe level decreases. This algorithms also forms nulls in the direction of interferes.

\section{REFERENCES}

[1] J. Bartolic, Z. Sipus, N. Herscovici, D. Bonefacic, and R. Zentner, "Planar and cylindrical microstrip patch antennas and array for wireless communications", $11^{\text {th }}$ Int. Conf. on Antenna Propag., Conference Publication, No 480, pp. 569-573, April 2001.

[2] L. C. Godara," Application of antenna arrays to mobile communication, Part I: Performance improvement feasibility, and system considerations", Proc. IEEE, vol. 85, no. 7, pp. 1031-1060, July 1997.

[3] L. C. Godara," Application of antenna arrays to mobile communication, Part II: Beam-forming, and direction-ofarrival considerations", Proc. IEEE, vol. 85, no. 8, pp. 1195-1245, August 1997.

[4] J.Litva and T.Kwok-Yeung Lo, " Digital Beamforming in Wireless Communication”, John Wiley and Sons, New York, 1996.

[5] J.H.Winters, "Smart Antennas for Wireless Systems", IEEE Personal Communications Magazine, pp. 23-27, February 1998. 